\title{
Communication \\ Overexpression of the MexXY Multidrug Efflux System Correlates with Deficient Pyoverdine Production in Pseudomonas aeruginosa
}

\author{
Kei Ikarashi ${ }^{1}$, Ryo Kutsuna ${ }^{2}$, Junko Tomida ${ }^{2}$, Yoshiaki Kawamura ${ }^{2}$ and Yuji Morita ${ }^{1, *(D)}$ \\ 1 Department of Infection Control Science, Meiji Pharmaceutical University, Tokyo 204-8588, Japan; \\ m196202@std.my-pharm.ac.jp \\ 2 Department of Microbiology, School of Pharmacy, Aichi Gakuin University, Aichi 464-8650, Japan; \\ kutsuna@dpc.agu.ac.jp (R.K.); jtomida@dpc.agu.ac.jp (J.T.); kawamura@dpc.agu.ac.jp (Y.K.) \\ * Correspondence: morita@my-pharm.ac.jp; Tel.: +81-42-495-8591
}

Citation: Ikarashi, K.; Kutsuna, R.; Tomida, J.; Kawamura, Y.; Morita, Y. Overexpression of the MexXY Multidrug Efflux System Correlates with Deficient Pyoverdine Production in Pseudomonas aeruginosa. Antibiotics 2021, 10, 658. https://doi.org/ 10.3390 /antibiotics 10060658

Academic Editor: Karl Hassan

Received: 19 April 2021

Accepted: 31 May 2021

Published: 31 May 2021

Publisher's Note: MDPI stays neutral with regard to jurisdictional claims in published maps and institutional affiliations.

Copyright: (c) 2021 by the authors. Licensee MDPI, Basel, Switzerland. This article is an open access article distributed under the terms and conditions of the Creative Commons Attribution (CC BY) license (https:// creativecommons.org/licenses/by/ $4.0 /)$.

\begin{abstract}
Multidrug-resistant Pseudomonas aeruginosa poses a serious problem due to hospital- and healthcare-associated infections. A major drug resistance mechanism of $P$. aeruginosa involves active efflux via resistance nodulation cell division (RND)-type multidrug efflux pumps of which MexXY is increasingly recognized as a primary determinant of aminoglycoside resistance in P. aeruginosa. MexXY overexpression is often observed in drug-resistant $P$. aeruginosa clinical isolates. MexXY deficiency increased pyoverdine production in all four P. aeruginosa strains we tested. MexXYoverproducing multidrug-resistant $P$. aeruginosa PA7 exhibited the greatest effect among the strains. Complementation with a MexXY-expressing plasmid restored low-level pyoverdine production in a MexXY-deficient P. aeruginosa mutant from PA7, indicating that MexXY expression decreases pyoverdine production. Because P. aeruginosa produces pyoverdine to acquire iron, MexXY-deficient mutants might be more susceptible to iron deficiency than MexXY-producing strains or might require extra iron. High-risk clones of multidrug-resistant $P$. aeruginosa reportedly tend to be MexXY overproducers but defective pyoverdine producers. This study suggests that $P$. aeruginosa reduces production of a virulence factor after acquiring a drug resistance factor.
\end{abstract}

Keywords: Pseudomonas aeruginosa; MexXY multidrug efflux system; pyoverdine production

\section{Introduction}

Pseudomonas aeruginosa is a known opportunistic pathogen and a major threat in hospital and healthcare-associated environments [1]. Infections caused by P. aeruginosa are often difficult to treat; inappropriate chemotherapy readily selects multidrug-resistant $P$. aeruginosa strains against which very few agents are effective [2,3]. A major factor in the prominence of $P$. aeruginosa as a pathogen is its intrinsic resistance to various antibacterial agents [2,3]. One of most important chromosomally encoded antimicrobial resistance factors in P. aeruginosa is resistance nodulation cell-division (RND)-type multidrug efflux pumps $[2,4]$. Among these pumps, the MexXY system is the only significant determinant of efflux-mediated aminoglycoside resistance in P. aeruginosa [5]. In addition to aminoglycosides, MexXY mediates resistance to other clinically relevant drugs such as cefepime, ciprofloxacin, tigecycline, azithromycin, and colistin [5,6].

Worldwide epidemic outbreaks of infection with highly drug-resistant $P$. aeruginosa are often associated with various so-called international high-risk clones [7], many of which harbor chromosomal mutations that promote drug resistance mechanisms, such as MexXY overproduction [8]. These clones also often produce lower amounts of pyoverdine in vitro, which has been proposed as a potential biomarker [9]. Pyoverdines that facilitate acute infections by pseudomonads include fluorescent siderophores, which specifically chelate $\mathrm{Fe}^{3+}$ with high affinity [10]. However, the types of gene mutations that contribute 
to defective pyoverdine production in highly multidrug-resistant $P$. aeruginosa clinical isolates remain unknown, as no major differences in pyoverdine gene clusters have been identified [11]. In this study, we conducted a detailed examination of the effect of MexXY on the production of pyoverdine in P. aeruginosa.

\section{Results}

During antimicrobial susceptibility tests of broth microdilution MIC methods (e.g., [12]) we found that strain PA7 $\triangle$ mexXY-oprA mutant were more yellow-green in color than those of the PA7 parent strain, which are highly multidrug resistant [13]. Therefore, we quantitatively examined pyoverdine production by four $P$. aeruginosa strains in comparison with the corresponding mexXY-deficient mutants (Figure 1). Of note, in our pyoverdine production assay system, fluorescence emission from PAO1 $\Delta p v d A$ [14] was almost negligible compared with the parent strain, PAO1 (data not shown). PA7 and K2153, a pan-aminoglycoside-resistant strain [15], exhibited markedly defective pyoverdine activity compared with PAO1, a drug-sensitive strain [15], whereas NCGM2. S1, a highly multidrugresistant strain [16], exhibited slightly but reproducibly lower pyoverdine production than strain PAO1 (Figure 1). Of note, both PA7 and K2153 are mexXY overproducers, whereas PAO1 and NCGM2. S1 are not [12,15].
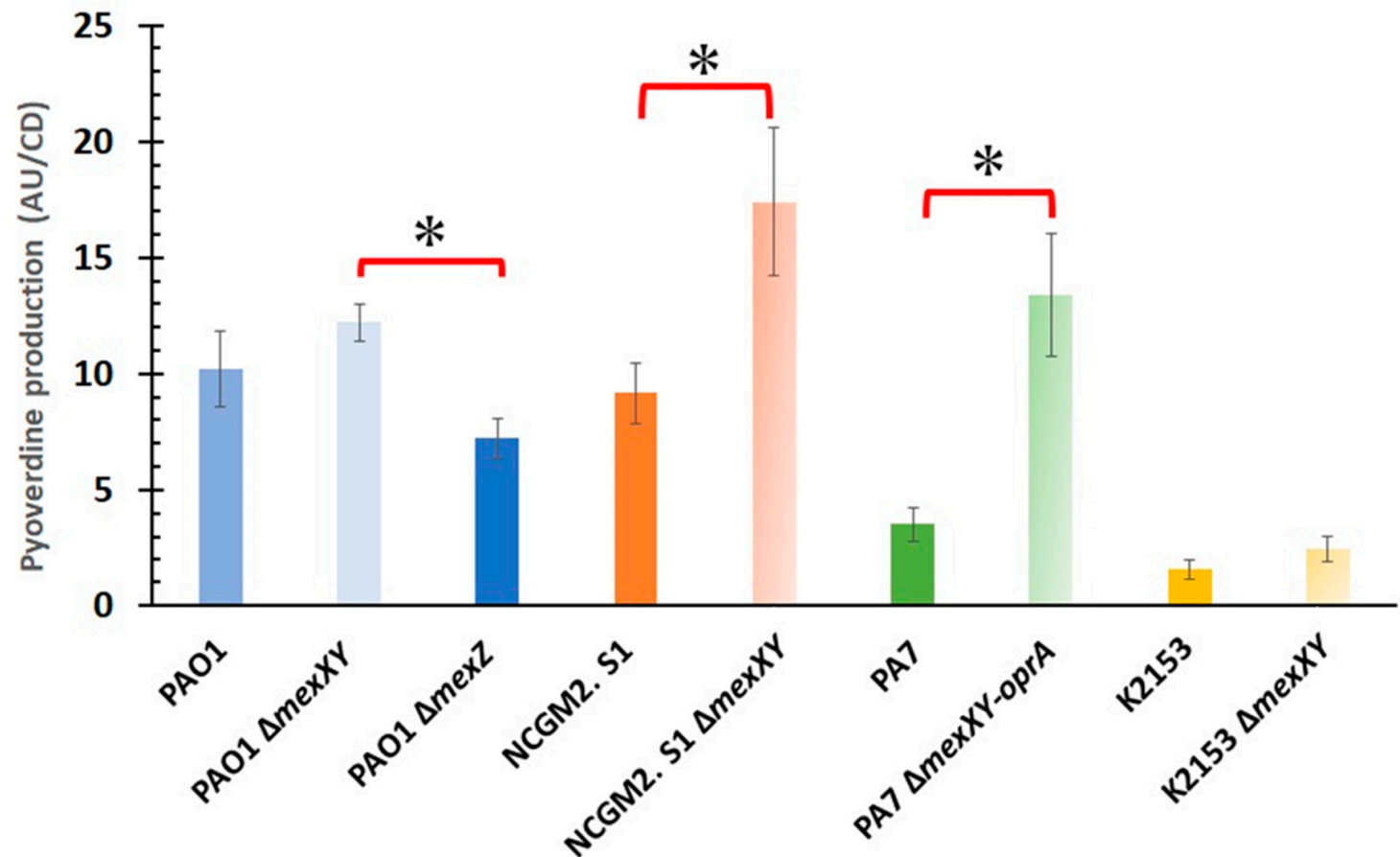

Figure 1. The MexXY multidrug efflux system decreased pyoverdine production in P. aeruginosa. Pyoverdine production was calculated by dividing the fluorescence value (arbitrary units, AU) by cell density (CD). The error range shown in the bar graph is the standard error $(n=5) .{ }^{*}$ Indicates $p<0.05$.

In all four strains examined, pyoverdine production increased due to MexXY deficiency (Figure 1). In particular, deficiency had the greatest impact on pyoverdine production in PA7 (3.8-fold increase), whereas MexXY deficiency was associated with a 1.2-fold increase in PAO1, a 1.9-fold increase in NCGM. 2 S1, and a 1.6-fold increase in K2153. In addition, deletion of mexZ, a local repressor gene of mexXY [17,18], resulted in a 1.7-fold decrease in pyoverdine production in strain PAO1 (Figure 1). Statistically significant differences in pyoverdine production were observed between PAO1 $\Delta$ mexXY and PAO1 $\Delta m e x Z$ ( $p$-value: $0.024[<0.05])$, between NCGM2. S1 and NCGM2. S1 $\Delta$ mexXY ( $p$-value: 0.032 $[<0.05])$, and between PA7 and PA7 $\Delta$ mexXY-oprA (p-value: $0.008[<0.05])$. No significant 
difference was observed, however, between K2153 and K2153 $\Delta$ mexXY ( $p$-value: 0.55 [>0.05]).

To confirm that the phenotypic changes due to mexXY deletion were actually due to loss of function of MexXY, we examined pyoverdine production by the complemented strain in which a mexXY-oprA expression plasmid was introduced into PA7 $\Delta m e x X Y$-oprA, in comparison with the negative control strain (Figure 2). Upon addition of isopropyl $\beta$-D-1-thiogalactopyranoside (IPTG) to induce plasmid-driven mexXY-oprA expression, the mexXY-oprA-expressing strain produced 2.7-fold more pyoverdine than the negative control strain ( $p$ value: $0.008[<0.05]$ ). In contrast, without IPTG, no significant difference in pyoverdine production was observed between the strains ( $p$ value: 0.30 [>0.05]).

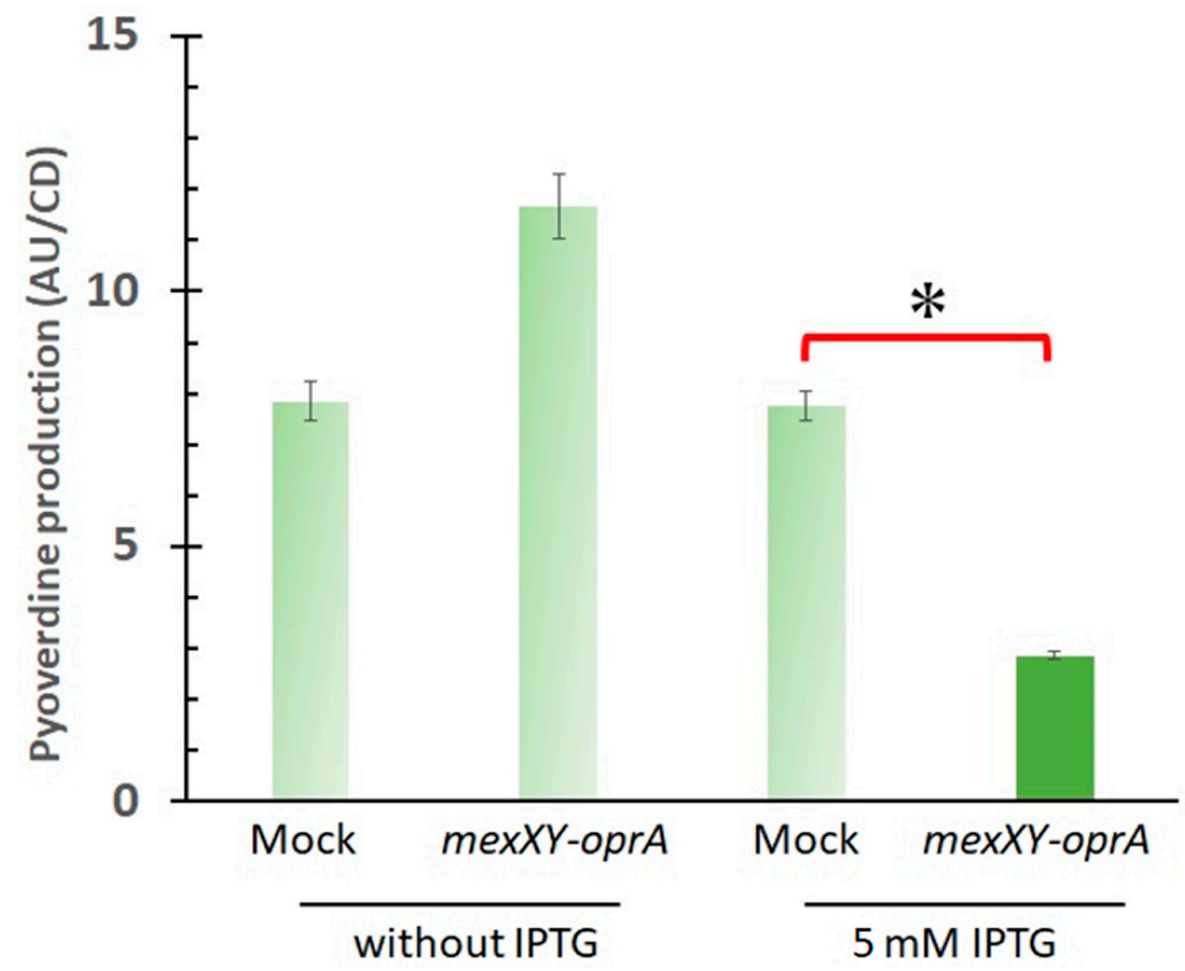

Figure 2. IPTG-induced mexXY-oprA expression decreased pyoverdine production in P. aeruginosa. Pyoverdine production was calculated by dividing the fluorescence value (arbitrary units, AU) by cell density (CD). Mock and mexXY-oprA on the horizontal axis indicate two complemented strains: PA7 $\Delta$ mexXY-oprA attB::pYM101 and PA7 $\Delta$ mexXY-oprA attB::pYM101-mexXY-oprA, respectively (Table 1). The error ranges shown in the bar graph are the standard error $(n=5) .{ }^{*}$ Indicates $p<0.05$. Cultures were supplemented with $5 \mathrm{mM}$ IPTG by addition to Pseudomonas agar F to drive the T7(A1/04/03) promoter expression system fully [12,19].

\section{Discussion}

The results of this study suggest that the MexXY multidrug efflux system decreases pyoverdine production in $P$. aeruginosa, indicating that MexXY-producing P. aeruginosa cells require less iron than MexXY-deficient cells, because P. aeruginosa requires pyoverdine for survival when iron concentrations become low [20]. The promotion of mexXY expression under conditions of oxidative stress is similar to the case of $P$. aeruginosa infection of chronically inflamed lungs of cystic fibrosis (CF) patients [21], which also induces siderophore biosynthesis genes [22], possibly due to oxidative inactivation of the Fur- $\mathrm{Fe}^{2+}$ complex [23]. A further study is thus necessary to elucidate the molecular mechanisms in more detail. A whole genome analyses of the studied P. aeruginosa strains can be one approach to use which might shed light and allow to get a broad picture.

Another possibility is that pyoverdine could be a substrate of MexXY, rather than MexXY decreasing pyoverdine production. The approach used for examining pyover- 
dine production does not rule out this possibility, since cells are taken from solid media, suspended in solution and then pyoverdine in the solution measured fluorometrically. Pyoverdine exported by cells on the solid media would presumably diffuse into the agar and thus might not be associated with cells collected for the assay although preliminary results from liquid culture supports the agar results in this study (Figure S1). If MexXY promotes pyoverdine efflux, mexXY deletion mutants could have higher cytosolic pyoverdine concentrations than the parental strains and carry more pyoverdine into the assay. The results would be more convincing if the cells were grown in liquid media and the spent media was assayed for pyoverdine concentration. It is also noteworthy that the buffer used for the pyoverdine assay contain the only contaminated iron. The absorbance spectra of free pyoverdine and iron-loaded pyoverdine are different. The maximum absorbance of iron-loaded pyoverdine is $400 \mathrm{~nm}$ (as used for excitation in the assay), but the maximum absorbance for unloaded pyoverdine is lower and $400 \mathrm{~nm}$ would only hit the shoulder. Therefore, having the only contaminated iron in the buffer might also impact the results of the assay.

Defective pyoverdine production is a biomarker of high epidemic risk P. aeruginosa clones [9], and multidrug resistant $P$. aeruginosa clinical isolates [5], including high epidemic risk clones [8], often overexpress mexXY. Decreased pyoverdine production could be, at least in part, due to mexXY overexpression in P. aeruginosa clinical isolates. We cannot rule out the possibility that other factors also contribute to defective pyoverdine production in $P$. aeruginosa clinical isolates. For example, mexXY expression does not appear to be the primary reason for defective pyoverdine production in P. aeruginosa K2153, a panaminoglycoside-resistant clinical isolate obtained from a CF patient (Figure 1). This study provides experimental evidence that upregulated expression of a drug-resistance factor leads to decreased production of a virulence factor in P. aeruginosa.

\section{Materials and Methods}

\subsection{Pseudomonas aeruginosa Strains and Growth Conditions}

Pseudomonas aeruginosa strains used in this study are listed in Table 1. Bacteria were grown in an Air-Jacketed Incubator IC802 (Yamato Scientific Co., Ltd., Tokyo, Japan) at $37^{\circ} \mathrm{C}$ under aerobic conditions, as previously described [12]. Unless otherwise indicated, bacteria were cultured using lysogeny broth, Lennox (LB)-agar prepared fresh from $1.0 \%$ Bacto $^{\mathrm{TM}}$ Tryptone (Becton, Dickinson and Company, Franklin Lakes, NJ, USA), 0.5\% Bacto $^{\mathrm{TM}}$ yeast extract (Becton, Dickinson and Company, Franklin Lakes, NJ, USA), and $0.5 \% \mathrm{NaCl}$ (FUJIFILM Wako Pure Chemical Corp., Osaka, Japan). Pseudomonas agar $\mathrm{F}$ was prepared from $2.0 \%$ Bacto $^{\mathrm{TM}}$ proteose peptone no. 3 (Becton, Dickinson and Company, Franklin Lakes, NJ, USA), 1.0\% Bacto ${ }^{\mathrm{TM}}$ Casitone (Becton, Dickinson and Company, Franklin Lakes, NJ, USA), 1.0\% glycerol (Nacalai Tesque Inc., Kyoto, Japan), $0.15 \% \mathrm{~K}_{2} \mathrm{HPO}_{4}$ (FUJIFILM Wako Pure Chemical Corp., Osaka, Japan), $0.073 \% \mathrm{MgSO}_{4} \cdot 7 \mathrm{H}_{2} \mathrm{O}$ (FUJIFILM Wako Pure Chemical Corp.), and 1.5\% agar (FUJIFILM Wako Pure Chemical Corp.), and used as a solid medium in STAR SDish9015 ver.2 petri dishes (Rikaken Co., Ltd., Nagoya, Japan) for assays of pyoverdine production. Bacterial growth was quantified by measuring the optical density at $600 \mathrm{~nm}\left(\mathrm{OD}_{600}\right)$ using a WPA CO8000 Cell Density Meter (Biochrom Ltd., Cambridge, UK).

\subsection{Assay of Pyoverdine Production}

Pyoverdine production was assayed according to a previously reported method [9]. Bacteria grown overnight at $37^{\circ} \mathrm{C}$ were suspended in $0.85 \%$ sterilized $\mathrm{NaCl}$ using a sterile wooden-axis cotton swab (Eiken Chemical Co., Ltd., Tokyo, Japan) and diluted to a final $\mathrm{OD}_{600}$ of 0.001 . Next, $100 \mu \mathrm{L}$ of the diluted culture was plated uniformly onto Pseudomonas $\mathrm{F}$ agar using a spreading stick and incubated at $37^{\circ} \mathrm{C}$ for $14 \mathrm{~h}$. Colonies of P. aeruginosa grown on the agar were suspended in $0.85 \%$ sterilized $\mathrm{NaCl}$ using a sterile wooden-axis cotton swab. After the cell density $\left(\mathrm{OD}_{600}\right)(\mathrm{A})$ was measured, the suspension was centrifuged at $23{ }^{\circ} \mathrm{C}$ and $4000 \times g$ for $10 \mathrm{~min}$. Each of three $200-\mu \mathrm{L}$ aliquots from the supernatant was 
transferred into each of three wells of a 96-well plate (Costar ${ }^{\circledR}$ no lid black, flat bottom, nontreated polystyrene (Corning Inc., Corning, NY, USA). The fluorescence emission for each well was measured (B) at an excitation wavelength of $400 \mathrm{~nm}$ and an emission wavelength of $460 \mathrm{~nm}$ using a SpectraMax iD3 multi-mode microplate reader (Molecular Devices, LLC, San Jose, CA, USA). Pyoverdine production was calculated by dividing (B) by (A). Each experiment was performed independently at least five times. When necessary, Pseudomonas agar F was supplemented with 5 mM IPTG (FUJIFILM Wako Pure Chemical Corp.).

Table 1. Pseudomonas aeruginosa strains used in this study.

\begin{tabular}{ccc}
\hline Lab Stock & Strain (=Co-Identical Strain) & Reference \\
\hline IMPU 1 & PAO1 (=K767 or PAGU 974) & {$[15]$} \\
IMPU 2 & NCGM2. S1 (=PAGU 1606) & {$[16]$} \\
IMPU 9 & PAO1 $\Delta$ mexXY (=K1525, PAGU 975) & {$[15]$} \\
IMPU 10 & NCGM2. S1 $\Delta$ mexXY (=PAGUg1659) & {$[12]$} \\
IMPU 17 & PAO1 $\Delta$ mexZ (=K2415, PAGUg1659) & {$[17]$} \\
IMPU 21 & PA7 (=PAGU 1498) & {$[13]$} \\
IMPU 29 & K2153 (=PAGU 1741) & {$[15]$} \\
IMPU 44 & PA7 $\Delta$ mexXY-oprA (=PAGUg1565) \\
IMPU 45 & K2153 $\Delta$ mexXY (=PAGUg1857) & {$[12]$} \\
IMPU 53 & PA7 $\Delta$ mexXY-oprA attB::pYM101 (=PAGUg1632) & {$[15]$} \\
IMPU 54 & PA7 $\Delta$ mexXY-oprA attB::pYM101-mexXY-oprA (=PAGUg1633) & {$[12]$} \\
IMPU 61 & PAO1 & {$[12]$} \\
IMPU 62 & PAO1 0 pvdA & {$[14]$} \\
*Co-identical &
\end{tabular}

* Co-identical strain is defined as a strain when stocked in the previous lab(s).

Statistical analyses were performed using the R software version 4.0.4 (https: / / www. r-project.org/) with the Wilcoxon rank test or Steel-Dwass test. A $p$-value of $<0.05$ was judged as indicating statistical significance.

Supplementary Materials: The following are available online at https:/ /www.mdpi.com/article/10 .3390 /antibiotics10060658/s1, Figure S1. The MexXY multidrug efflux system decreased pyoverdine production from liquid culture in P. aeruginosa.

Author Contributions: Conceptualization, Y.M.; methodology, Y.M.; formal analysis, K.I. and Y.M.; investigation, K.I. and Y.M.; resources, Y.K. and Y.M.; data curation, K.I. and Y.M.; writing-original draft preparation, Y.M.; writing—review and editing, Y.M.; visualization, K.I. and Y.M.; supervision, Y.M.; validation, R.K., J.T., K.I. and Y.M.; project administration, Y.M.; funding acquisition, Y.M. All authors have read and agreed to the published version of the manuscript.

Funding: This work was supported by JSPS KAKENHI Grant Number 19K07547 and was performed under the Research Program of "Dynamic Alliance for Open Innovation Bridging Human, Environment and Materials" in "Network Joint Research Center for Materials and Devices".

Institutional Review Board Statement: Not applicable.

Informed Consent Statement: Not applicable.

Acknowledgments: We thank Haruka Kumada (Meiji Pharmaceutical University, Japan), Yoshiaki Sakairi (Meiji Pharmaceutical University, Japan), Kotaro Suzuki (Meiji Pharmaceutical University, Japan), Shinya Suzuki (Aichi Gakuin University, Japan), and Tadashi Kumazawa (Aichi Gakuin University, Japan) for their contributions. We also thank Tohru Miyoshi-Akiyama (International Medical Center of Japan), R. Keith Poole (Queen's University, Canada), Paul H. Roy (Université Laval, Canada), and Paolo Visca (Roma Tre University, Italy) for providing bacterial strains.

Conflicts of Interest: The authors declare no conflict of interest.

\section{References}

1. Gellatly, S.L.; Hancock, R.E. Pseudomonas aeruginosa: New insights into pathogenesis and host defenses. Pathog. Dis. 2013, 67, 159-173. [CrossRef]

2. Poole, K. Pseudomonas aeruginosa: Resistance to the Max. Front. Microbiol. 2011, 2, 65. [CrossRef] [PubMed] 
3. Morita, Y.; Tomida, J.; Kawamura, Y. Resistance and Response to Anti-Pseudomonas Agents and Biocides. In Pseudomonas, 1st ed.; Ramos, J.L., Goldberg, J., Filloux, A., Eds.; Springer Science and Business Media: Dordrecht, The Netherlands, 2014 ; pp. 173-187.

4. Li, X.-Z.; Plésiat, P.; Nikaido, H. The Challenge of Efflux-Mediated Antibiotic Resistance in Gram-Negative Bacteria. Clin. Microbiol. Rev. 2015, 28, 337-418. [CrossRef]

5. Morita, Y.; Tomida, J.; Kawamura, Y. MexXY multidrug efflux system of Pseudomonas aeruginosa. Front. Microbiol. 2012,3 , 408. [CrossRef] [PubMed]

6. Puja, H.; Bolard, A.; Noguès, A.; Plésiat, P.; Jeannot, K. The Efflux Pump MexXY/OprM Contributes to the Tolerance and Acquired Resistance of Pseudomonas aeruginosa to Colistin. Antimicrob. Agents Chemother. 2020, 64, e02033-19. [CrossRef]

7. Horcajada, J.P.; Montero, M.; Oliver, A.; Sorlí, L.; Luque, S.; Gómez-Zorrilla, S.; Benito, N.; Grau, S. Epidemiology and Treatment of Multidrug-Resistant and Extensively Drug-Resistant Pseudomonas aeruginosa Infections. Clin. Microbiol. Rev. 2019, 32. [CrossRef]

8. Cabot, G.; Ocampo-Sosa, A.A.; Domínguez, M.A.; Gago, J.F.; Juan, C.; Tubau, F.; Rodríguez, C.; Moyà, B.; Peña, C.; Martínez, L.M.; et al. Genetic Markers of Widespread Extensively Drug-Resistant Pseudomonas aeruginosa High-Risk Clones. Antimicrob. Agents Chemother. 2012, 56, 6349-6357. [CrossRef]

9. Mulet, X.; Cabot, G.; Ocampo-Sosa, A.A.; Domínguez, M.A.; Zamorano, L.; Juan, C.; Tubau, F.; Rodríguez, C.; Moyà, B.; Peña, C.; et al. Biological Markers of Pseudomonas aeruginosa Epidemic High-Risk Clones. Antimicrob. Agents Chemother. 2013, 57, $5527-5535$. [CrossRef]

10. Cornelis, P.; Dingemans, J. Pseudomonas aeruginosa adapts its iron uptake strategies in function of the type of infections. Front. Cell. Infect. Microbiol. 2013, 3, 75. [CrossRef]

11. Hwang, W.; Yoon, S.S. Virulence Characteristics and an Action Mode of Antibiotic Resistance in Multidrug-Resistant Pseudomonas aeruginosa. Sci. Rep. 2019, 9, 1-15. [CrossRef] [PubMed]

12. Morita, Y.; Tomida, J.; Kawamura, Y. Primary mechanisms mediating aminoglycoside resistance in the multidrug-resistant Pseudomonas aeruginosa clinical isolate PA7. Microbiology 2012, 158, 1071-1083. [CrossRef] [PubMed]

13. Roy, P.H.; Tetu, S.G.; Larouche, A.; Elbourne, L.; Tremblay, S.; Ren, Q.; Dodson, R.; Harkins, D.; Shay, R.; Watkins, K.; et al. Complete Genome Sequence of the Multiresistant Taxonomic Outlier Pseudomonas aeruginosa PA7. PLoS ONE 2010, 5, e8842. [CrossRef]

14. Imperi, F.; Putignani, L.; Tiburzi, F.; Ambrosi, C.; Cipollone, R.; Ascenzi, P.; Visca, P. Membrane-association determinants of the $\omega$-amino acid monooxygenase PvdA, a pyoverdine biosynthetic enzyme from Pseudomonas aeruginosa. Microbiology 2008, 154, 2804-2813. [CrossRef] [PubMed]

15. Sobel, M.L.; McKay, G.A.; Poole, K. Contribution of the MexXY Multidrug Transporter to Aminoglycoside Resistance in Pseudomonas aeruginosa Clinical Isolates. Antimicrob. Agents Chemother. 2003, 47, 3202-3207. [CrossRef]

16. Miyoshi-Akiyama, T.; Kuwahara, T.; Tada, T.; Kitao, T.; Kirikae, T. Complete Genome Sequence of Highly Multidrug-Resistant Pseudomonas aeruginosa NCGM2.S1, a Representative Strain of a Cluster Endemic to Japan. J. Bacteriol. 2011, 193, 7010. [CrossRef]

17. Morita, Y.; Sobel, M.L.; Poole, K. Antibiotic Inducibility of the MexXY Multidrug Efflux System of Pseudomonas aeruginosa: Involvement of the Antibiotic-Inducible PA5471 Gene Product. J. Bacteriol. 2006, 188, 1847-1855. [CrossRef]

18. Guénard, S.; Muller, C.; Monlezun, L.; Benas, P.; Broutin, I.; Jeannot, K.; Plésiat, P. Multiple Mutations Lead to MexXY-OprMDependent Aminoglycoside Resistance in Clinical Strains of Pseudomonas aeruginosa. Antimicrob. Agents Chemother. 2013, 58, 221-228. [CrossRef]

19. Morita, Y.; Nakashima, K.-I.; Nishino, K.; Kotani, K.; Tomida, J.; Inoue, M.; Kawamura, Y. Berberine Is a Novel Type Efflux Inhibitor Which Attenuates the MexXY-Mediated Aminoglycoside Resistance in Pseudomonas aeruginosa. Front. Microbiol. 2016, 7, 1223. [CrossRef] [PubMed]

20. Ringel, M.T.; Brüser, T. The biosynthesis of pyoverdines. Microb. Cell 2018, 5, 424-437. [CrossRef]

21. Fraud, S.; Poole, K. Oxidative Stress Induction of the MexXY Multidrug Efflux Genes and Promotion of Aminoglycoside Resistance Development in Pseudomonas aeruginosa. Antimicrob. Agents Chemother. 2010, 55, 1068-1074. [CrossRef] [PubMed]

22. Palma, M.; DeLuca, D.; Worgall, S.; Quadri, L.E.N. Transcriptome Analysis of the Response of Pseudomonas aeruginosa to Hydrogen Peroxide. J. Bacteriol. 2004, 186, 248-252. [CrossRef] [PubMed]

23. Imlay, J.A. The molecular mechanisms and physiological consequences of oxidative stress: Lessons from a model bacterium. Nat. Rev. Genet. 2013, 11, 443-454. [CrossRef] [PubMed] 